\title{
Facing and managing natural disasters in the Sporades islands, Greece
}

\author{
P. Karanikola ${ }^{1}$, T. Panagopoulos ${ }^{2}$, S. Tampakis ${ }^{1}$, M. I. Karantoni ${ }^{1}$, and G. Tsantopoulos ${ }^{1}$ \\ ${ }^{1}$ Department of Forestry and Management of the Environment and Natural Resources, Democritus University of Thrace, 193, \\ Pantazidou str., 68200 Orestiada, Greece \\ ${ }^{2}$ Research Centre for Spatial and Organizational Dynamics (CIEO), University of Algarve, Gambelas Campus, 8000 Faro, \\ Portugal
}

Correspondence to: T. Panagopoulos (tpanago@ualg.pt)

Received: 1 October 2013 - Published in Nat. Hazards Earth Syst. Sci. Discuss.: 19 November 2013

Revised: - - Accepted: 14 March 2014 - Published: 25 April 2014

\begin{abstract}
The region of the Sporades islands located in central Greece is at the mercy of many natural phenomena, such as earthquakes due to the marine volcano Psathoura and the rift of Anatolia, forest fires, floods, landslides, storms, hail, snowfall and frost. The present work aims at studying the perceptions and attitudes of the residents regarding how they face and manage natural disasters. A positive public response during a hazard crisis depends not only upon the availability and good management of a civil defense plan but also on the knowledge and perception of the possible hazards by the local population. It is important for the stakeholders to know what the citizens expect so that the necessary structures can be developed in the phase of preparation and organization. The residents were asked their opinion about what they think should be done by the stakeholders after a catastrophic natural disaster, particularly about the immediate response of stakeholders and their involvement and responsibilities at different, subsequent intervals of time following the disaster. The residents were also asked about the most common disasters that happen in their region and about the preparation activities of the stakeholders.
\end{abstract}

\section{Introduction}

A natural disaster is a physical event of extraordinary dimension that people cannot predict or control (Djelante, 2012). It is the most rapid, instantaneous and long-range conflict of the natural environment with the socioeconomic system and the human society (Mercer, 2009; Cutter et al., 2013). Thus, the natural process becomes a "natural hazard" as soon as human beings, infrastructure or other forms of tangible or intangible capital are threatened or destroyed. The losses concern both the animate and inanimate potential of human society, both intangibly and materially (Varnes, 1988; Raschky, 2008).

A disaster, either natural (earthquake, fire, etc.) or caused by humans (war conflict, nuclear accident), shapes the human and natural environment and disrupts and affects the operation of the region at an economic and social level depending, of course, on its degree and extent (Becker et al., 2013; Yellman and Murray, 2013).

According to Miletti (1999), the disasters are a "forecasted" result that comes from the interaction of three main systems: (a) the physical environment, (b) the demographic and socioeconomic environment, and (c) the structured environment (residences, buildings and infrastructures). It has been noted that the event and the results of a disaster are due to critical correlations between the three systems, from which they acquire more complex textures (Haimes, 2012).

The link between development and disasters is well known; unfortunately, despite the modern evolution of technology and the progress of science in general, natural disasters affect the daily lives of people, disturb the smooth operation of society and constitute a permanent threat (Otero and Marti, 1995; Stenchion, 1997; Pelling, 2003a; McEntire, 2004; UNDP, 2004; Wisner et al., 2004).

The extreme events can even be devastating for developing countries that have less capacity to adapt (Mendelsohn and Dinar, 1999; Ravindranath and Sathaye, 2002; Winkler, 
2005; IPCC, 2007a), but the effects generally influence both developed and developing or underdeveloped regions.

Generally, major natural disasters are the consequence of a natural hazard, which passes from the stage of probability to an active phase; consequently, they have serious implications for the economic, developmental and environmental sectors. Equally considerable are the problems that they create at the social, political and cultural level (Cutter et al., 2003), as well as in the administrative sector. Depending on the size and type of disaster, the period following the destruction can be of long or short term. There is a global concern that natural disasters are becoming more frequent, deadly and costly; they are also more complex, and the impacts to society and the environment are increasingly more intertwined (Khan, 2012). For this reason, the treatment and management of natural disasters is one of the biggest problems of survival that currently engages humans because they constitute a milestone in the human consciousness and dramatically affect the flow of their lives (Drabek, 1986; Starmer, 1996; Sterlacchini et al., 2007; EM-DAT, 2010; EM-DAT, 2013; Germanwatch, 2010; IFRC, 2010; Maplecroft, 2010; UNISDR, 2011; UNU-EHS, 2011). It is very important for the authorities and the stakeholders to know what the population expects from them after a large-scale natural disaster. This information can be very useful to an organization and help to properly structure the necessary preparations.

One of the major approaches of disaster risk reduction is through pragmatic disaster risk management planning (Salter, 1997; Christoplos et al., 2001). Disaster management and community planning via public participation have become top priority for the authorities, stakeholders and organizations in many countries all over the world such as in the USA (Pearce, 2003, Haimes, 2012), El Salvador (Bowman and White, 2012), Australia and New Zealand (Gero et al., 2011; Djalante, 2012; Becker et al., 2012, 2013) China (Ye et al., 2012, Shi et al., 2012) and Iran (Amini Hosseini et al., 2009). In Europe there have already been some papers about this subject (Van Assche et al., 2011; Escuder-Bueno et al., 2012; Alexander, 2013), but no relevant studies have been conducted so far in Greece.

The present work aims at studying the perceptions and attitudes of the residents in the Sporades islands, Greece, about the management of natural disasters and the expectations of the authorities and the relevant stakeholders in the first crucial hours, days or weeks following a catastrophic event.

\section{Research methodology}

The research was conducted with the application of a face-toface structured questionnaire. The research area of this paper was the islands of the Northern Sporades. The statistical populations of the Alonissos, Skiathos and Skopelos were 2160, 5788 and 4098 residents, respectively. Layered, random sampling was used as the sampling method. Geographical strati- fication layers were also used. The estimate of proportion $P$ of the population was a weighted analogy of samples. The size of each sample was taken so that the number of units of the population belonging to each layer was as follows:

$$
\bar{P}=\frac{1}{N}\left(N_{1} \bar{P}_{1}+N_{2} \bar{P}_{2}+\ldots+N_{L} \bar{P}_{L}\right)=\frac{1}{N} \sum_{k=1}^{L}\left(N_{k} \bar{P}_{k}\right),
$$

where $L$ indicates the number of layers, $N_{k}$ the total number of sample units in the layer $k(k=1, \ldots, L), N$ total number of sample units in the population $(n=N 1, N L)$, and $P_{k}$ estimated proportion in layer $k$.

The estimated standard error of the proportion is

$$
s_{\overline{\mathrm{p}}}=\sqrt{\frac{1}{N^{2}} \sum_{k=1}^{L}\left(N_{k}^{2} \frac{\bar{P}_{k}\left(1-\bar{P}_{k}\right)}{n_{k}-1}\right)},
$$

where $n_{k}$ indicates the sample size in layer $k$. The estimates, as with simple random sampling, can be made separately for each layer since each layer was taken as a simple random sample (Daoutopoulos, 1994). The results of each layer are presented separately (for each island).

In order to determine the sample size, pre-sampling was used. The data were collected through random, personal interviews, and 66 questionnaires (12 from the municipality of Alonissos, 30 from the municipality of Skiathos and 24 from the municipality of Skopelos) were collected in total.

The determination of the total sample size for the variables, analogue distribution, is given by the following formula:

$$
\begin{aligned}
& n=\frac{N t^{2} \sum_{k=1}^{L} N_{k} \bar{P}_{k}\left(1-\bar{P}_{k}\right)}{N^{2} e^{2}+t^{2} \sum_{h=1}^{L} N_{k} \bar{P}_{k}\left(1-\bar{P}_{k}\right)} \\
& =\frac{12919 \times 1.96^{2} \times(2425 \times 0.5 \times 0.5+5788 \times 0.5 \times 0.5+4706 \times 0.5 \times 0.5)}{12919^{2} \times 0.05^{2}+1.96^{2} \times(2425 \times 0.5 \times 0.5+5788 \times 0.5 \times 0.5+4706 \times 0.5 \times 0.5)} \\
& =373.0665 \cong 373,
\end{aligned}
$$

where $N$ denotes the total number of sample units of all layers, $N_{k}$ denotes the total number of sample units in the layer $k, P_{k}$ denotes the estimated proportion in layer $k, t$ denotes the value of Student's distribution for probability $(1-\alpha)=95 \%$ and $n-1$ degree of freedom, and $e$ denotes the maximum admissible difference between the sampling medium and unknown average population. We accept that in the case of proportions it is 0.05 , that is, $5 \%$. 
Table 1. Sociodemographic profile of the residents in the Sporades islands ( $s_{\mathrm{p}}$ : Standard error of proportion).

\begin{tabular}{|c|c|c|c|}
\hline & & $p(\%)$ & $s_{\mathrm{p}}$ \\
\hline \multirow[t]{2}{*}{ Gender } & Male & 52.0 & 0.0260 \\
\hline & Female & 48.0 & 0.0260 \\
\hline \multirow[t]{5}{*}{ Age } & $18-30$ & 23.3 & 0.0216 \\
\hline & $31-40$ & 33.2 & 0.0243 \\
\hline & $41-50$ & 23.1 & 0.0219 \\
\hline & $51-60$ & 10.5 & 0.0159 \\
\hline & $>60$ & 11.0 & 0.0160 \\
\hline \multirow[t]{4}{*}{ Marital status } & Unmarried & 31.6 & 0.0241 \\
\hline & Married & 58.4 & 0.0256 \\
\hline & Divorced & 4.3 & 0.0104 \\
\hline & Widowed & 5.6 & 0.0120 \\
\hline \multirow[t]{5}{*}{ Childhood } & Without children & 39.9 & 0.0254 \\
\hline & One child & 15.6 & 0.0188 \\
\hline & Two children & 26.8 & 0.0230 \\
\hline & Three children & 12.6 & 0.0172 \\
\hline & More than three children & 5.1 & 0.0114 \\
\hline \multirow[t]{8}{*}{ Educational level } & Without primary school & 7.0 & 0.0135 \\
\hline & Primary school & 9.6 & 0.0149 \\
\hline & Secondary school & 15.0 & 0.0184 \\
\hline & Technical school & 4.3 & 0.0105 \\
\hline & Upper secondary school & 34.9 & 0.0246 \\
\hline & Technological education & 10.7 & 0.0161 \\
\hline & University & 13.4 & 0.0175 \\
\hline & Post-graduation & 5.1 & 0.0114 \\
\hline \multirow[t]{8}{*}{ Profession } & Private employee & 22.3 & 0.0214 \\
\hline & Public servants & 21.7 & 0.0212 \\
\hline & Self-employed & 20.1 & 0.0207 \\
\hline & Students & 4.3 & 0.0104 \\
\hline & Unemployed & 7.2 & 0.0133 \\
\hline & Housewives & 8.0 & 0.0140 \\
\hline & Farmers or stock breeders & 4.0 & 0.0101 \\
\hline & Pensioners & 12.3 & 0.0169 \\
\hline \multirow[t]{9}{*}{ Annual income } & $<5000$ euro & 20.4 & 0.0206 \\
\hline & 5000-10 000 euro & 15.5 & 0.0188 \\
\hline & $10001-15000$ euro & 15.0 & 0.0185 \\
\hline & $15001-20000$ euro & 19.0 & 0.0202 \\
\hline & $20001-25000$ euro & 8.0 & 0.0140 \\
\hline & $25001-30000$ euro & 4.0 & 0.0102 \\
\hline & $30001-40000$ euro & 4.8 & 0.0111 \\
\hline & $>40000$ euro & 4.3 & 0.0105 \\
\hline & No answer & 8.8 & 0.0148 \\
\hline
\end{tabular}

The total size of the sample is distributed in different layers according to the size of each layer.

$$
\begin{aligned}
& n_{k}=\frac{N_{k} n}{N}= \\
& n_{1} \frac{2425 \times 373}{12919}=70.0276 \cong 70 \\
& n_{2} \frac{5788 \times 373}{12919}=167.1421 \cong 167 \\
& n_{3} \frac{4706 \times 373}{12919}=135.8968 \cong 136
\end{aligned}
$$

The questionnaire is not limited to a single variable estimation of the population, as it contains more variables. So an estimate of the sample size is required for each of the variables. If the estimated sample sizes are similar, and their size is within the numerical possibilities of the sampling, then the sample size is selected as the maximum. In this way the variable that changes the most is estimated with the desired precision while remaining more accurate than originally specified (Matis, 2001). The variable that gives the largest sample size is the one that refers to the gender of respondents.

A total of 70 questionnaires were collected in the municipality of Alonissos, as well as 167 in the municipality of Skiathos and 136 in the municipality of Skopelos. Data collection took place in 2009. Personal interviews were conducted to supplement the questionnaire. They were randomly selected from the citizens of the municipality.

\section{Results}

The demographic attributes of the residents who participated in the research are given in Table 1. The majorities of the participants were men (52\%), graduates of upper school education $(34.5 \%)$, married $(58.4 \%)$, without children $(39.9 \%)$ and private employees $(22.3 \%)$. Seventy percent of the participants declared an income of less than 20 thousand euro. The largest age group of the participants $\left(33.2 \%, s_{\mathrm{p}}=0.0243\right)$ was $31-40$ years old.

\subsection{Actions to be taken, chronologically, after a natural disaster}

Natural disasters affect human society and have diverse consequences, such as loss of human life, economic damage, damage to the residential system, psychological consequences, destruction of monuments and exhibits in museums, and even political consequences (Papadopoulos, 2000). Following an unexpected natural disaster, the first problem that is faced is the identification of the extent of the disaster based on the disorder of the population, the transportation, the operation of organisms and the flow of information. Having precisely determined the region that was affected by the disaster, direct priority is given to the search and rescue of survivors. The functionality and capacity of hospitals should be checked, and those that have not been affected by the disaster should be provided with additional staff and first-aid supplies. Particular attention should be provided to individuals that are not self-sufficient, such as young children, the elderly, the disabled, etc. Additionally, rescue teams and supplies should be concentrated in regions where they can be best used, and are absolutely necessary. Furthermore, during the management of a disaster, it is very important to determine the number of affected people versus the number of visitors and curious onlookers in the disaster zone, a process that is not easy in the chaotic situation that follows a disaster. 
Table 2. Residents' opinions and attitudes about what is most necessary to be done, chronologically, after an extreme natural disaster.

\begin{tabular}{|c|c|c|c|c|c|c|c|c|c|c|c|}
\hline & & $0-3 \mathrm{~h}$ & $3-6 \mathrm{~h}$ & $6-12 \mathrm{~h}$ & $12-24 \mathrm{~h}$ & Next day & 3-7 days & Next week & $2-4$ weeks & Next months & Next years \\
\hline \multirow[t]{2}{*}{ Food supply } & $p$ & $41.0 \%$ & $22.5 \%$ & $18.2 \%$ & $9.7 \%$ & $8.0 \%$ & $0.3 \%$ & $0.3 \%$ & & & \\
\hline & $s_{\mathrm{p}}$ & 0.0254 & 0.0216 & 0.0199 & 0.0153 & 0.0134 & 0.0027 & 0.0027 & & & \\
\hline \multirow[t]{2}{*}{ Supply of clean water } & $p$ & $53.6 \%$ & $26.3 \%$ & $8.8 \%$ & $3.5 \%$ & $7.5 \%$ & $0.3 \%$ & & & & \\
\hline & $s_{\mathrm{p}}$ & 0.0258 & 0.0228 & 0.0148 & 0.0095 & 0.0129 & 0.0027 & & & & \\
\hline \multirow[t]{2}{*}{ Medical care of the injured } & $p$ & $77.2 \%$ & $16.4 \%$ & $4.8 \%$ & $1.3 \%$ & $0.3 \%$ & & & & & \\
\hline & $s_{\mathrm{p}}$ & 0.0216 & 0.0190 & 0.0111 & 0.0060 & 0.0027 & & & & & \\
\hline \multirow[t]{2}{*}{ Official information } & $p$ & $36.5 \%$ & $19.8 \%$ & $9.9 \%$ & $14.7 \%$ & $17.7 \%$ & $0.8 \%$ & $0.5 \%$ & & & \\
\hline & $s_{\mathrm{p}}$ & 0.0249 & 0.0204 & 0.0154 & 0.0184 & 0.0190 & 0.0046 & 0.0038 & & & \\
\hline \multirow[t]{2}{*}{ Temporary accommodation for the affected } & $p$ & $14.5 \%$ & $21.4 \%$ & $19.6 \%$ & $27.6 \%$ & $15.3 \%$ & $1.6 \%$ & & & & \\
\hline & $s_{\mathrm{p}}$ & 0.0183 & 0.0212 & 0.0203 & 0.0231 & 0.0184 & 0.0065 & & & & \\
\hline \multirow[t]{2}{*}{ Psychological support of the injured } & $p$ & $20.9 \%$ & $20.6 \%$ & $11.0 \%$ & $13.4 \%$ & $25.5 \%$ & $5.9 \%$ & $1.9 \%$ & $0.3 \%$ & $0.5 \%$ & \\
\hline & $s_{\mathrm{p}}$ & 0.0207 & 0.0210 & 0.0161 & 0.0177 & 0.0226 & 0.0121 & 0.0070 & 0.0027 & 0.0038 & \\
\hline \multirow[t]{2}{*}{ Search and rescue of victims } & $p$ & $78.3 \%$ & $10.5 \%$ & $5.6 \%$ & $1.9 \%$ & $3.2 \%$ & $0.3 \%$ & $0.3 \%$ & & & \\
\hline & $s_{\mathrm{p}}$ & 0.0214 & 0.0157 & 0.0120 & 0.0070 & 0.0092 & 0.0027 & 0.0027 & & & \\
\hline \multirow[t]{2}{*}{ Transportation of citizens to a safer place } & $p$ & $53.4 \%$ & $24.7 \%$ & $11.5 \%$ & $4.8 \%$ & $5.1 \%$ & & $0.5 \%$ & & & \\
\hline & $s_{\mathrm{p}}$ & 0.0254 & 0.0220 & 0.0165 & 0.0111 & 0.0114 & & 0.0038 & & & \\
\hline \multirow[t]{2}{*}{ Recognition and identification of victims } & $p$ & $15.8 \%$ & $15.3 \%$ & $19.6 \%$ & $11.8 \%$ & $30.6 \%$ & $5.6 \%$ & $1.3 \%$ & & & \\
\hline & $s_{\mathrm{p}}$ & 0.0190 & 0.0185 & 0.0205 & 0.0167 & 0.0236 & 0.0119 & 0.0059 & & & \\
\hline \multirow[t]{2}{*}{ Respectful handling of human remains } & $p$ & $33.0 \%$ & $12.1 \%$ & $12.6 \%$ & $12.9 \%$ & $22.0 \%$ & $4.8 \%$ & $2.1 \%$ & $0.5 \%$ & & \\
\hline & $s_{\mathrm{p}}$ & 0.0235 & 0.0169 & 0.0170 & 0.0173 & 0.0214 & 0.0111 & 0.0075 & 0.0038 & & \\
\hline \multirow[t]{2}{*}{ Making lists of the injured, missing and dead } & $p$ & $19.0 \%$ & $12.3 \%$ & $11.8 \%$ & $10.7 \%$ & $36.7 \%$ & $6.4 \%$ & $2.4 \%$ & $0.5 \%$ & & \\
\hline & $s_{\mathrm{p}}$ & 0.0202 & 0.0170 & 0.0166 & 0.0159 & 0.0249 & 0.0127 & 0.0079 & 0.0038 & & \\
\hline \multirow[t]{2}{*}{ Occupation of children with various activities } & $p$ & $12.1 \%$ & $6.7 \%$ & $9.1 \%$ & $9.9 \%$ & $22.0 \%$ & $20.6 \%$ & $11.5 \%$ & $2.4 \%$ & $4.0 \%$ & $1.6 \%$ \\
\hline & $s_{\mathrm{p}}$ & 0.0165 & 0.0129 & 0.0148 & 0.0153 & 0.0215 & 0.0209 & 0.0164 & 0.0080 & 0.0101 & 0.0065 \\
\hline \multirow[t]{2}{*}{ Guarding property against theft } & $p$ & $31.6 \%$ & $13.4 \%$ & $11.3 \%$ & $13.4 \%$ & $22.0 \%$ & $4.6 \%$ & $2.1 \%$ & $1.3 \%$ & $0.3 \%$ & \\
\hline & $s_{\mathrm{p}}$ & 0.0237 & 0.0175 & 0.0161 & 0.0175 & 0.0215 & 0.0107 & 0.0075 & 0.0059 & 0.0027 & \\
\hline \multirow[t]{2}{*}{ Existence of people to inform and lead the affected people } & $p$ & $46.9 \%$ & $20.4 \%$ & $8.6 \%$ & $7.5 \%$ & $11.3 \%$ & $3.5 \%$ & $1.3 \%$ & $0.5 \%$ & & \\
\hline & $s_{\mathrm{p}}$ & 0.0251 & 0.0208 & 0.0145 & 0.0136 & 0.0163 & 0.0094 & 0.0059 & 0.0038 & & \\
\hline \multirow[t]{2}{*}{ Timely assessment of the damages } & $p$ & $6.4 \%$ & $6.2 \%$ & $6.4 \%$ & $8.6 \%$ & $26.8 \%$ & $21.5 \%$ & $18.0 \%$ & $5.1 \%$ & $1.1 \%$ & \\
\hline & $s_{\mathrm{p}}$ & 0.0125 & 0.0125 & 0.0127 & 0.0145 & 0.0227 & 0.0210 & 0.0199 & 0.0114 & 0.0053 & \\
\hline \multirow[t]{2}{*}{ Temporary repair of the damage } & $p$ & $4.6 \%$ & $3.2 \%$ & $6.2 \%$ & $4.0 \%$ & $15.8 \%$ & $28.2 \%$ & $21.2 \%$ & $10.7 \%$ & $5.4 \%$ & $0.8 \%$ \\
\hline & $s_{\mathrm{p}}$ & 0.0108 & 0.0090 & 0.0125 & 0.0102 & 0.0190 & 0.0234 & 0.0212 & 0.0160 & 0.0116 & 0.0046 \\
\hline \multirow[t]{2}{*}{ Assessment of damages to infrastructures } & $p$ & $4.0 \%$ & $6.4 \%$ & $4.0 \%$ & $3.2 \%$ & $19.8 \%$ & $18.2 \%$ & $25.2 \%$ & $13.9 \%$ & $4.3 \%$ & $0.8 \%$ \\
\hline & $s_{\mathrm{p}}$ & 0.0102 & 0.0122 & 0.0101 & 0.0091 & 0.0207 & 0.0200 & 0.0225 & 0.0180 & 0.0104 & 0.0046 \\
\hline \multirow[t]{2}{*}{ Assessment of damages in private buildings } & $p$ & $2.9 \%$ & $6.2 \%$ & $1.6 \%$ & $3.5 \%$ & $16.6 \%$ & $18.8 \%$ & $28.2 \%$ & $11.8 \%$ & $8.0 \%$ & $2.4 \%$ \\
\hline & $s_{\mathrm{p}}$ & 0.0088 & 0.0121 & 0.0065 & 0.0095 & 0.0193 & 0.0203 & 0.0232 & 0.0168 & 0.0139 & 0.0079 \\
\hline \multirow[t]{2}{*}{ Providing economic support to those affected } & $p$ & $3.8 \%$ & $1.9 \%$ & $1.1 \%$ & $3.5 \%$ & $11.0 \%$ & $14.2 \%$ & $24.9 \%$ & $19.6 \%$ & $15.8 \%$ & $4.3 \%$ \\
\hline & $s_{\mathrm{p}}$ & 0.0098 & 0.0070 & 0.0053 & 0.0095 & 0.0163 & 0.0181 & 0.0224 & 0.0205 & 0.0188 & 0.0104 \\
\hline \multirow[t]{2}{*}{ Economic support for businesses to start working again } & $p$ & $1.6 \%$ & $0.0 \%$ & $4.0 \%$ & $3.2 \%$ & $5.9 \%$ & $8.8 \%$ & $24.1 \%$ & $21.4 \%$ & $24.4 \%$ & $6.4 \%$ \\
\hline & $s_{\mathrm{p}}$ & 0.0065 & 0.0000 & 0.0102 & 0.0092 & 0.0122 & 0.0147 & 0.0222 & 0.0213 & 0.0222 & 0.0126 \\
\hline \multirow[t]{2}{*}{ General organizational plan of the region } & $p$ & $13.4 \%$ & $0.5 \%$ & $1.3 \%$ & $1.6 \%$ & $6.2 \%$ & $5.9 \%$ & $15.3 \%$ & $12.6 \%$ & $31.6 \%$ & $11.5 \%$ \\
\hline & $s_{\mathrm{p}}$ & 0.0173 & 0.0038 & 0.0060 & 0.0065 & 0.0124 & 0.0122 & 0.0184 & 0.0172 & 0.0239 & 0.0165 \\
\hline
\end{tabular}

Mutual help is also of vital importance because the survivors should share their food, shelter and means of available transport (Lekkas, 2000).

The period following a disaster corresponds to the shortterm efforts to address it in the emergency management and the relief stage. It generally takes about 2 days, and some researchers suggest a sequence of priorities that should be used to address the emergency period in the most effective way (Alexander, 2013). However, because it is difficult to simultaneously address all needs in such a short period of time, citizens were asked to evaluate the range of importance of each need. Thus, from the relative question (Table 2), it was found that the residents of the Sporades believe that the first operations in the $3 \mathrm{~h}$ following a natural disaster are the search and rescue of victims $(78.3 \%)$, the medical care of the injured $(77.2 \%)$, the supplying of clean water $(53.6 \%)$ and the transportation of citizens to a safer place $(53.4 \%)$. In the same period, other priorities for the citizens include identification of a person to inform and lead the affected people $(46.9 \%)$, food supply $(41.0 \%)$, provision of official information $(36.5 \%)$, respectful handling of human remains $(33.0 \%)$, guarding property against theft $(31.6 \%)$ and giving psychological support $(20.9 \%)$. However, the citizens consider that those actions, while still important, can be implemented with a $12 \mathrm{~h}$ delay. During the next 12 to $24 \mathrm{~h}$, the residents believe that temporary accommodation to the affected people is necessary $(27.6 \%)$. On the third day, the citizens believe that these tasks can take place: the listing of the injured, missing and dead $(36.7 \%)$; the recognition and identification of victims $(30.6 \%)$; the timely assessment of 
Table 3. Residents' opinions about the responsibilities that stakeholders have after an extreme natural disaster.

\begin{tabular}{|c|c|c|c|c|c|c|c|c|c|c|c|}
\hline & & 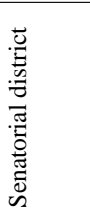 & 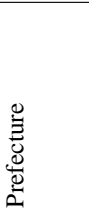 & 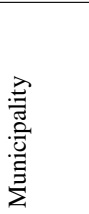 & $\begin{array}{l}\stackrel{\circlearrowright}{0} \\
\stackrel{0}{0} \\
\infty \\
0 \\
0\end{array}$ & 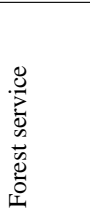 & $\stackrel{\mathscr{0}}{: 0}$ & 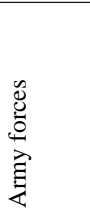 & 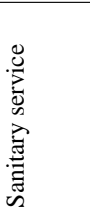 & 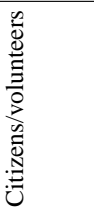 & 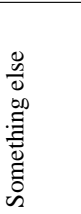 \\
\hline Food supply & $\begin{array}{l}p \\
s_{\mathrm{p}}\end{array}$ & $\begin{array}{l}24.9 \% \\
0.0224\end{array}$ & $\begin{array}{l}30.3 \% \\
0.0237\end{array}$ & $\begin{array}{l}86.6 \% \\
0.0177\end{array}$ & $\begin{array}{l}1.3 \% \\
0.0059\end{array}$ & $\begin{array}{l}0.3 \% \\
0.0027\end{array}$ & $\begin{array}{l}1.1 \% \\
0.0053\end{array}$ & $\begin{array}{l}17.2 \% \\
0.0193\end{array}$ & $\begin{array}{l}3.8 \% \\
0.0098\end{array}$ & $\begin{array}{l}32.2 \% \\
0.0242\end{array}$ & $\begin{array}{l}2.4 \% \\
0.0079\end{array}$ \\
\hline Supply of clean water & $\begin{array}{l}p \\
s_{\mathrm{p}}\end{array}$ & $\begin{array}{l}19.0 \% \\
0.0202\end{array}$ & $\begin{array}{l}26.5 \% \\
0.0228\end{array}$ & $\begin{array}{l}83.9 \% \\
0.0190\end{array}$ & $\begin{array}{l}9.4 \% \\
0.0143\end{array}$ & $\begin{array}{l}1.6 \% \\
0.0065\end{array}$ & $\begin{array}{l}0.8 \% \\
0.0046\end{array}$ & $\begin{array}{l}15.5 \% \\
0.0187\end{array}$ & $\begin{array}{l}7.2 \% \\
0.0135\end{array}$ & $\begin{array}{l}24.4 \% \\
0.0223\end{array}$ & $\begin{array}{l}2.7 \% \\
0.0083\end{array}$ \\
\hline Medical care of the injured & $\begin{array}{l}p \\
s_{\mathrm{p}}\end{array}$ & $\begin{array}{l}5.9 \% \\
0.0122\end{array}$ & $\begin{array}{l}6.7 \% \\
0.0129\end{array}$ & $\begin{array}{l}14.2 \% \\
0.0181\end{array}$ & $\begin{array}{l}4.6 \% \\
0.0108\end{array}$ & $\begin{array}{l}0.5 \% \\
0.0038\end{array}$ & $\begin{array}{l}1.3 \% \\
0.0060\end{array}$ & $\begin{array}{l}20.9 \% \\
0.0210\end{array}$ & $\begin{array}{l}86.3 \% \\
0.0178\end{array}$ & $\begin{array}{l}19.3 \% \\
0.0205\end{array}$ & $\begin{array}{l}1.9 \% \\
0.0070\end{array}$ \\
\hline Official information & $\begin{array}{l}p \\
s_{\mathrm{p}}\end{array}$ & $\begin{array}{l}58.5 \% \\
0.0241\end{array}$ & $\begin{array}{l}33.5 \% \\
0.0245\end{array}$ & $\begin{array}{l}42.9 \% \\
0.0257\end{array}$ & $\begin{array}{l}13.1 \% \\
0.0173\end{array}$ & $\begin{array}{l}7.2 \% \\
0.0134\end{array}$ & $\begin{array}{l}22.5 \% \\
0.0209\end{array}$ & $\begin{array}{l}1.9 \% \\
0.0070\end{array}$ & $\begin{array}{l}2.1 \% \\
0.0075\end{array}$ & $\begin{array}{l}0.5 \% \\
0.0038\end{array}$ & $\begin{array}{l}2.4 \% \\
0.0079\end{array}$ \\
\hline Temporary accommodation for the affected & $\begin{array}{l}p \\
s_{\mathrm{p}}\end{array}$ & $\begin{array}{l}23.6 \% \\
0.0220\end{array}$ & $\begin{array}{l}31.4 \% \\
0.0235\end{array}$ & $\begin{array}{l}71.9 \% \\
0.0231\end{array}$ & $\begin{array}{l}3.8 \% \\
0.0098\end{array}$ & $\begin{array}{l}3.5 \% \\
0.0095\end{array}$ & $\begin{array}{l}3.5 \% \\
0.0095\end{array}$ & $\begin{array}{l}42.4 \% \\
0.0252\end{array}$ & $\begin{array}{l}5.9 \% \\
0.0122\end{array}$ & $\begin{array}{l}24.4 \% \\
0.0222\end{array}$ & $\begin{array}{l}2.9 \% \\
0.0087\end{array}$ \\
\hline Psychological support of the injured & $\begin{array}{l}p \\
s_{\mathrm{p}}\end{array}$ & $\begin{array}{l}9.9 \% \\
0.0154\end{array}$ & $\begin{array}{l}12.9 \% \\
0.0171\end{array}$ & $\begin{array}{l}24.4 \% \\
0.0223\end{array}$ & $\begin{array}{l}3.8 \% \\
0.0099\end{array}$ & $\begin{array}{l}1.6 \% \\
0.0065\end{array}$ & $\begin{array}{l}4.6 \% \\
0.0108\end{array}$ & $\begin{array}{l}4.8 \% \\
0.0111\end{array}$ & $\begin{array}{l}67.8 \% \\
0.0238\end{array}$ & $\begin{array}{l}34.8 \% \\
0.0246\end{array}$ & $\begin{array}{l}9.9 \% \\
0.0152\end{array}$ \\
\hline Search and rescue of victims & $\begin{array}{l}p \\
s_{\mathrm{p}}\end{array}$ & $\begin{array}{l}5.4 \% \\
0.0116\end{array}$ & $\begin{array}{l}9.9 \% \\
0.0153\end{array}$ & $\begin{array}{l}29.0 \% \\
0.0230\end{array}$ & $\begin{array}{l}77.2 \% \\
0.0216\end{array}$ & $\begin{array}{l}27.9 \% \\
0.0233\end{array}$ & $\begin{array}{l}53.1 \% \\
0.0259\end{array}$ & $\begin{array}{l}63.5 \% \\
0.0248\end{array}$ & $\begin{array}{l}16.9 \% \\
0.0194\end{array}$ & $\begin{array}{l}34.3 \% \\
0.0245\end{array}$ & $\begin{array}{l}2.1 \% \\
0.0075\end{array}$ \\
\hline Transportation of citizens to a safer place & $\begin{array}{l}p \\
s_{\mathrm{p}}\end{array}$ & $\begin{array}{l}9.4 \% \\
0.0149\end{array}$ & $\begin{array}{l}11.5 \% \\
0.0162\end{array}$ & $\begin{array}{l}51.7 \% \\
0.0245\end{array}$ & $\begin{array}{l}44.8 \% \\
0.0251\end{array}$ & $\begin{array}{l}24.1 \% \\
0.0219\end{array}$ & $\begin{array}{l}55.5 \% \\
0.0257\end{array}$ & $\begin{array}{l}54.4 \% \\
0.0259\end{array}$ & $\begin{array}{l}12.1 \% \\
0.0167\end{array}$ & $\begin{array}{l}29.2 \% \\
0.0232\end{array}$ & $\begin{array}{l}2.7 \% \\
0.0083\end{array}$ \\
\hline Recognition and identification of victims & $\begin{array}{l}p \\
s_{\mathrm{p}}\end{array}$ & $\begin{array}{l}2.1 \% \\
0.0075\end{array}$ & $\begin{array}{l}2.1 \% \\
0.0075\end{array}$ & $\begin{array}{l}20.9 \% \\
0.0210\end{array}$ & $\begin{array}{l}9.9 \% \\
0.0154\end{array}$ & $\begin{array}{l}4.6 \% \\
0.0107\end{array}$ & $\begin{array}{l}41.0 \% \\
0.0240\end{array}$ & $\begin{array}{l}10.2 \% \\
0.0156\end{array}$ & $\begin{array}{l}33.8 \% \\
0.0245\end{array}$ & $\begin{array}{l}23.3 \% \\
0.0205\end{array}$ & $\begin{array}{l}11.8 \% \\
0.0166\end{array}$ \\
\hline Respectful handling of human remains & $\begin{array}{l}p \\
s_{\mathrm{p}}\end{array}$ & $\begin{array}{l}33.2 \% \\
0.0237\end{array}$ & $\begin{array}{l}18.5 \% \\
0.0201\end{array}$ & $\begin{array}{l}33.0 \% \\
0.0242\end{array}$ & $\begin{array}{l}20.6 \% \\
0.0210\end{array}$ & $\begin{array}{l}16.4 \% \\
0.0192\end{array}$ & $\begin{array}{l}33.2 \% \\
0.0243\end{array}$ & $\begin{array}{l}22.3 \% \\
0.0216\end{array}$ & $\begin{array}{l}51.7 \% \\
0.0257\end{array}$ & $\begin{array}{l}16.6 \% \\
0.0193\end{array}$ & $\begin{array}{l}15.5 \% \\
0.0187\end{array}$ \\
\hline Making lists of the injured, missing and dead & $\begin{array}{l}p \\
s_{\mathrm{p}}\end{array}$ & $\begin{array}{l}8.0 \% \\
0.0140\end{array}$ & $\begin{array}{l}10.7 \% \\
0.0158\end{array}$ & $\begin{array}{l}59.8 \% \\
0.0252\end{array}$ & $\begin{array}{l}10.7 \% \\
0.0158\end{array}$ & $\begin{array}{l}4.8 \% \\
0.0111\end{array}$ & $\begin{array}{l}38.1 \% \\
0.0235\end{array}$ & $\begin{array}{l}7.0 \% \\
0.0131\end{array}$ & $\begin{array}{l}22.8 \% \\
0.0216\end{array}$ & $\begin{array}{l}16.9 \% \\
0.0180\end{array}$ & $\begin{array}{l}4.6 \% \\
0.0108\end{array}$ \\
\hline Occupation of children with various activities & $\begin{array}{l}p \\
s_{\mathrm{p}}\end{array}$ & $\begin{array}{l}11.0 \% \\
0.0161\end{array}$ & $\begin{array}{l}16.4 \% \\
0.0190\end{array}$ & $\begin{array}{l}42.9 \% \\
0.0257\end{array}$ & $\begin{array}{l}2.9 \% \\
0.0087\end{array}$ & $\begin{array}{l}0.5 \% \\
0.0038\end{array}$ & $\begin{array}{l}4.3 \% \\
0.0105\end{array}$ & $\begin{array}{l}1.9 \% \\
0.0070\end{array}$ & $\begin{array}{l}10.2 \% \\
0.0157\end{array}$ & $\begin{array}{l}60.3 \% \\
0.0254\end{array}$ & $\begin{array}{l}13.4 \% \\
0.0172\end{array}$ \\
\hline Guarding property against theft & $\begin{array}{l}p \\
s_{\mathrm{p}}\end{array}$ & $\begin{array}{l}3.2 \% \\
0.0091\end{array}$ & $\begin{array}{l}2.4 \% \\
0.0080\end{array}$ & $\begin{array}{l}17.2 \% \\
0.0191\end{array}$ & $\begin{array}{l}3.8 \% \\
0.0099\end{array}$ & $\begin{array}{l}4.6 \% \\
0.0108\end{array}$ & $\begin{array}{l}89.3 \% \\
0.0161\end{array}$ & $\begin{array}{l}35.1 \% \\
0.0246\end{array}$ & $\begin{array}{l}0.8 \% \\
0.0046\end{array}$ & $\begin{array}{l}6.7 \% \\
0.0130\end{array}$ & $\begin{array}{l}1.1 \% \\
0.0054\end{array}$ \\
\hline People to inform and lead the affected people & $\begin{array}{l}p \\
s_{\mathrm{p}}\end{array}$ & $\begin{array}{l}13.9 \% \\
0.0178\end{array}$ & $\begin{array}{l}19.8 \% \\
0.0203\end{array}$ & $\begin{array}{l}61.4 \% \\
0.0243\end{array}$ & $\begin{array}{l}31.6 \% \\
0.0239\end{array}$ & $\begin{array}{l}23.6 \% \\
0.0218\end{array}$ & $\begin{array}{l}41.6 \% \\
0.0256\end{array}$ & $\begin{array}{l}29.2 \% \\
0.0233\end{array}$ & $\begin{array}{l}9.9 \% \\
0.0154\end{array}$ & $\begin{array}{l}48.3 \% \\
0.0257\end{array}$ & $\begin{array}{l}6.2 \% \\
0.0123\end{array}$ \\
\hline Timely assessment of the damages & $\begin{array}{l}p \\
s_{\mathrm{p}}\end{array}$ & $\begin{array}{l}54.2 \% \\
0.0252\end{array}$ & $\begin{array}{l}49.9 \% \\
0.0258\end{array}$ & $\begin{array}{l}49.6 \% \\
0.0258\end{array}$ & $\begin{array}{l}7.8 \% \\
0.0138\end{array}$ & $\begin{array}{l}4.3 \% \\
0.0105\end{array}$ & $\begin{array}{l}5.1 \% \\
0.0114\end{array}$ & $\begin{array}{l}1.1 \% \\
0.0053\end{array}$ & $\begin{array}{l}1.1 \% \\
0.0053\end{array}$ & $\begin{array}{l}0.8 \% \\
0.0046\end{array}$ & $\begin{array}{l}6.4 \% \\
0.0125\end{array}$ \\
\hline Temporary repair of the damage & $\begin{array}{l}p \\
s_{\mathrm{p}}\end{array}$ & $\begin{array}{l}57.4 \% \\
0.0251\end{array}$ & $\begin{array}{l}55.8 \% \\
0.0258\end{array}$ & $\begin{array}{l}47.7 \% \\
0.0260\end{array}$ & $\begin{array}{l}2.4 \% \\
0.0079\end{array}$ & $\begin{array}{l}2.9 \% \\
0.0087\end{array}$ & $\begin{array}{l}2.4 \% \\
0.0079\end{array}$ & $\begin{array}{l}5.1 \% \\
0.0112\end{array}$ & $\begin{array}{l}1.1 \% \\
0.0053\end{array}$ & $\begin{array}{l}4.8 \% \\
0.0111\end{array}$ & $\begin{array}{l}4.8 \% \\
0.0110\end{array}$ \\
\hline Assessment of damages to infrastructures & $\begin{array}{l}p \\
s_{\mathrm{p}}\end{array}$ & $\begin{array}{l}57.9 \% \\
0.0251\end{array}$ & $\begin{array}{l}55.5 \% \\
0.0257\end{array}$ & $\begin{array}{l}45.0 \% \\
0.0258\end{array}$ & $\begin{array}{l}4.0 \% \\
0.0102\end{array}$ & $\begin{array}{l}2.7 \% \\
0.0083\end{array}$ & $\begin{array}{l}4.8 \% \\
0.0110\end{array}$ & $\begin{array}{l}2.1 \% \\
0.0075\end{array}$ & $\begin{array}{l}0.0 \% \\
0.0000\end{array}$ & $\begin{array}{l}1.3 \% \\
0.0059\end{array}$ & $\begin{array}{l}5.1 \% \\
0.0113\end{array}$ \\
\hline Assessment of damages in private buildings & $\begin{array}{l}p \\
s_{\mathrm{p}}\end{array}$ & $\begin{array}{l}56.0 \% \\
0.0254\end{array}$ & $\begin{array}{l}53.1 \% \\
0.0258\end{array}$ & $\begin{array}{l}44.2 \% \\
0.0258\end{array}$ & $\begin{array}{l}4.0 \% \\
0.0102\end{array}$ & $\begin{array}{l}1.1 \% \\
0.0053\end{array}$ & $\begin{array}{l}5.4 \% \\
0.0116\end{array}$ & $\begin{array}{l}1.6 \% \\
0.0065\end{array}$ & $\begin{array}{l}0.5 \% \\
0.0038\end{array}$ & $\begin{array}{l}1.6 \% \\
0.0065\end{array}$ & $\begin{array}{l}6.4 \% \\
0.0125\end{array}$ \\
\hline Providing an economic support to those affected & $\begin{array}{l}p \\
s_{\mathrm{p}}\end{array}$ & $\begin{array}{l}75.1 \% \\
0.0224\end{array}$ & $\begin{array}{l}46.9 \% \\
0.0253\end{array}$ & $\begin{array}{l}33.0 \% \\
0.0240\end{array}$ & $\begin{array}{l}1.1 \% \\
0.0053\end{array}$ & $\begin{array}{l}1.1 \% \\
0.0053\end{array}$ & $\begin{array}{l}0.3 \% \\
0.0027\end{array}$ & $\begin{array}{l}0.8 \% \\
0.0046\end{array}$ & $\begin{array}{l}0.8 \% \\
0.0046\end{array}$ & $\begin{array}{l}5.4 \% \\
0.0117\end{array}$ & $\begin{array}{l}19.0 \% \\
0.0202\end{array}$ \\
\hline Economic support for businesses to start working again & $\begin{array}{l}p \\
s_{\mathrm{p}}\end{array}$ & $\begin{array}{l}75.9 \% \\
0.0222\end{array}$ & $\begin{array}{l}42.1 \% \\
0.0250\end{array}$ & $\begin{array}{l}25.7 \% \\
0.0224\end{array}$ & $\begin{array}{l}0.5 \% \\
0.0038\end{array}$ & $\begin{array}{l}0.5 \% \\
0.0038\end{array}$ & $\begin{array}{l}1.3 \% \\
0.0059\end{array}$ & $\begin{array}{l}0.0 \% \\
0.0000\end{array}$ & $\begin{array}{l}0.5 \% \\
0.0038\end{array}$ & $\begin{array}{l}1.9 \% \\
0.0070\end{array}$ & $\begin{array}{l}21.2 \% \\
0.0210\end{array}$ \\
\hline General organizational plan of the region & $\begin{array}{l}p \\
s_{\mathrm{p}}\end{array}$ & $\begin{array}{l}75.1 \% \\
0.0223\end{array}$ & $\begin{array}{l}44.8 \% \\
0.0248\end{array}$ & $\begin{array}{l}45.3 \% \\
0.0249\end{array}$ & $\begin{array}{l}9.7 \% \\
0.0152\end{array}$ & $\begin{array}{l}8.6 \% \\
0.0144\end{array}$ & $\begin{array}{l}8.8 \% \\
0.0146\end{array}$ & $\begin{array}{l}7.5 \% \\
0.0136\end{array}$ & $\begin{array}{l}4.8 \% \\
0.0111\end{array}$ & $\begin{array}{l}5.1 \% \\
0.0114\end{array}$ & $\begin{array}{l}8.0 \% \\
0.0140\end{array}$ \\
\hline
\end{tabular}

the damages $(26.8 \%)$; and the occupation of children with various activities $(22.0 \%)$.

In the second phase of rehabilitation that begins in the next 3-7 days (the medium term of recovery), the residents believe that it is necessary to start temporary repairs of the damage $(28.2 \%)$ while, for the next week, an assessment of damages in private buildings should be undertaken $(28.2 \%)$, along with the infrastructures $(25.2 \%)$ and the provision of economic support to those affected $(24.9 \%)$.

Finally, during the period of reconstruction in the upcoming months, the residents believe that it is necessary to design a regional organization plan $(31.6 \%)$ and to provide economic support for business redeployment (24.4\%). 
Table 4. Residents' opinions about the activities that happen in the Sporades islands that affect the consequences of floods.

\begin{tabular}{lcccccc}
\hline & & Very often & Usually & Sometimes & Rarely & Never \\
\hline Construction of flood protection works and preserving them & $p$ & $5.1 \%$ & $18.2 \%$ & $29.5 \%$ & $22.8 \%$ & $24.4 \%$ \\
& $s_{\mathrm{p}}$ & 0.0113 & 0.0196 & 0.0237 & 0.0216 & 0.0220 \\
\hline Fixing forest soils & $p$ & $3.2 \%$ & $18.0 \%$ & $29.8 \%$ & $22.0 \%$ & $27.1 \%$ \\
& $s_{\mathrm{p}}$ & 0.0091 & 0.0194 & 0.0237 & 0.0215 & 0.0228 \\
\hline Illegal occupation of streams and polders & $p$ & $30.3 \%$ & $24.7 \%$ & $29.2 \%$ & $9.1 \%$ & $6.7 \%$ \\
& $s \mathrm{p}$ & 0.0236 & 0.0223 & 0.0234 & 0.0148 & 0.0128 \\
\hline Construction of public roads with drainage systems & $p$ & $7.0 \%$ & $18.8 \%$ & $32.4 \%$ & $18.2 \%$ & $23.6 \%$ \\
& $s \mathrm{p}$ & 0.0131 & 0.0199 & 0.0243 & 0.0200 & 0.0218 \\
\hline
\end{tabular}

Table 5. Residents' opinions about the activities that take place in the Sporades islands regarding snow/frost consequences.

\begin{tabular}{lcccccc}
\hline & Very often & Usually & Sometimes & Rarely & Never \\
\hline Facing problems from ice and snow & $p$ & $13.7 \%$ & $20.1 \%$ & $32.7 \%$ & $22.0 \%$ & $11.5 \%$ \\
& $s_{\mathrm{p}}$ & 0.0173 & 0.0206 & 0.0236 & 0.0214 & 0.0165 \\
\hline $\begin{array}{l}\text { Control, limitation and exclusion (obligatory use of nonslip } \\
\text { chains) }\end{array}$ & $p$ & $6.2 \%$ & $16.4 \%$ & $28.4 \%$ & $30.8 \%$ & $18.2 \%$ \\
& $s_{\mathrm{p}}$ & 0.0123 & 0.0191 & 0.0234 & 0.0239 & 0.0198 \\
\hline Car movement on the roads when it is not absolutely neces- & $p$ & $12.9 \%$ & $25.7 \%$ & $29.8 \%$ & $22.5 \%$ & $9.1 \%$ \\
sary & & & & & & \\
& $s_{\mathrm{p}}$ & 0.0174 & 0.0227 & 0.0238 & 0.0214 & 0.0146 \\
\hline Closed roads due to cars blocking the road & $p$ & $5.9 \%$ & $8.6 \%$ & $30.3 \%$ & $41.0 \%$ & $14.2 \%$ \\
& $s_{\mathrm{p}}$ & 0.0120 & 0.0142 & 0.0229 & 0.0247 & 0.0180 \\
\hline
\end{tabular}

\subsection{Stakeholders' active involvement in disaster management}

Risk management is not the exclusive duty of one organization, but rather the result of the coordinated actions of several operators, where everyone has a specific role. Generally, the main responsibility of civil protection of the country falls on the Ministry of Interior along with the General Secretariat of Civil Protection, the fire service, the police and the forest service. The district, the prefecture and the municipality are responsible for the implementation of regional planning based on the available resources. Important roles are also played by non-governmental organizations and volunteers.

Table 3 presents the results of the questionnaire about the institution that should take the responsibilities after the outbreak of a major disaster. The inhabitants of the Sporades islands consider that the senatorial district should be responsible for providing economic support to help businesses start working again $(75.9 \%)$ as well as to provide economic support to those affected by the disaster $(75.1 \%)$. Further, they should also develop a general plan of regional regeneration $(75.1 \%)$, and provide official information $(58.5 \%)$, temporary damage repair $(57.4 \%)$ and damage assessment (54.2\%). The respondents believe that the municipality should be responsible for the supply of food $(86.6 \%)$ and water (83.9\%), for drawing up a list of names of the injured, missing and dead $(59.8 \%)$ and for leading and informing the affected citizens $(61.4 \%)$. The citizens believe that the fire service should be responsible for the search and rescue of victims $(77.2 \%)$, the armed forces should give temporary accommodation $(42.2 \%)$ and the police should maintain both public safety and public order $(89.3 \%)$, transport citizens to a safer place $(55.5 \%)$ and help in the recognition and identification of the victims (41\%). Finally, they believe that the sanitary service should be responsible for the medical care of the injured (86.3\%), for the psychological support of the injured $(67.8 \%)$, and respectful handling of human remains $(51.7 \%)$. Volunteers should be responsible for occupying the children with activities $(60.3 \%)$.

\subsection{Actions to confront natural disasters in the Sporades islands}

The region of Sporades is at the mercy of many natural phenomena; in most cases the effects of these phenomena are quite unfavorable to the residents of the islands. More analytically, the region is affected by earthquakes from nearby sources like the marine volcano Psathoura, as well as the rift of Anatolia that passes N-NW of Alonissos resulting in intense seismic activity and frequent earthquakes. A typical 
Table 6. Residents' opinions about the activities that take place in the Sporades islands regarding earthquakes consequences.

\begin{tabular}{|c|c|c|c|c|c|c|}
\hline & & Very often & Usually & Sometimes & Rarely & Never \\
\hline \multirow[t]{2}{*}{ New buildings checked for earthquake standards } & $p$ & $3.8 \%$ & $11.0 \%$ & $24.4 \%$ & $29.2 \%$ & $31.6 \%$ \\
\hline & $s_{\mathrm{p}}$ & 0.0098 & 0.0162 & 0.0221 & 0.0234 & 0.0241 \\
\hline \multirow[t]{2}{*}{ Information to the citizens about activities in case of an earthquake } & $p$ & $4.0 \%$ & $7.5 \%$ & $21.2 \%$ & $31.6 \%$ & $35.7 \%$ \\
\hline & $s_{\mathrm{p}}$ & 0.0102 & 0.0135 & 0.0212 & 0.0241 & 0.0245 \\
\hline \multirow[t]{2}{*}{ Infringement of construction licensing or illegal buildings } & $p$ & $31.4 \%$ & $26.0 \%$ & $21.7 \%$ & $13.1 \%$ & $7.8 \%$ \\
\hline & $s_{\mathrm{p}}$ & 0.0235 & 0.0227 & 0.0212 & 0.0175 & 0.0136 \\
\hline \multirow[t]{2}{*}{ Spreading information about forthcoming earthquakes } & $p$ & $22.0 \%$ & $16.6 \%$ & $22.3 \%$ & $23.3 \%$ & $15.8 \%$ \\
\hline & $s_{\mathrm{p}}$ & 0.0210 & 0.0192 & 0.0214 & 0.0214 & 0.0184 \\
\hline
\end{tabular}

example is the earthquake in 1986, which shook the earth for 40 days. Moreover, the last 10 years have seen the frequent occurrence of another type of phenomena, forest fires, which have destroyed important forest and agricultural lands. Soil erosion and landslides are subsequent hazards associated with forest wildfires (De Graff et al., 2013). Flooding is of low intensity at the study area and has only created minor problems, while landslides, heavy storms, hail, snowfall and frost are frequent and intense.

For the most common natural disasters that happen in their region (floods, snow, ice, earthquakes and forest fires), the residents were asked if they were aware of activities that may affect the danger or occurrence of these disasters. Table 4 presents the results regarding floods and landslides. The participants answered that the illegal occupation of streams and polders has a negative affect $(30.3 \%)$. Proper road drainage $(32.4 \%)$, stream restoration and slope stabilization $(29.8 \%)$ may decrease flood risk.

Regarding snow and frost (Table 5), the citizens responded that they sometimes faced problems from such hazards $(32.7 \%)$, and they sometimes drove their cars on the roads when it was not absolutely necessary (29.8\%). Moreover, citizens $(41 \%)$ rarely experienced closed roads due to cars blocking the road, and $30.8 \%$ of the citizens indicated that control, limitation and exclusion rarely occur due to the obligatory use of nonslip chains.

Table 6 presents the results that the respondents provided about earthquakes. Infringement of construction licensing or illegal buildings $(31.4 \%)$ is the most frequently reported problem, while leaked uncontrolled information about forthcoming earthquakes was rare $(23.3 \%)$. Furthermore, citizens answered that they were rarely or never informed of the activities that should be taken in the case of an earthquake $(35.7 \%)$. In the opinion of the respondents, there is no checking of earthquake standards for new buildings (31.6\%).

Finally, regarding forest fires, the residents believe that citizens very often participate in the suppression of forest fires $(41.8 \%)$, that the fire service usually conducts frequent patrols in the forests $(33.8 \%)$ and that there are sufficient fire guardrooms during the fire season $(26 \%)$. Also, the respon- dents have the opinion that there is illegal occupation on forestlands on a very frequent basis $(27.9 \%)$, while the citizens rarely participate in reforestations $(25.2 \%)$. Moreover, they answered that the causes of forest fires are the following: not cleaning dry vegetation off of property $(32.6 \%)$, lit cigarettes thrown from cars $(35.4 \%)$, the removal of vegetation from the edges of roads and paths $(30.8 \%)$ and the burning of agricultural remains during the dry season $(29.8 \%)$ (Table 7).

\section{Discussion and conclusions}

Statements made by residents implied that many of them were aware of the probability of risks from natural hazards in their region. Natural hazards pose threats to vulnerable infrastructure, visitors and the public (Whitworth and May, 2006). Hazard assessment and risk governance has become increasingly politicized and controversial (Armas and Avram, 2009). For this reason risk reduction is important and can be achieved when public participation is integrated into disaster management planning and community planning (Pearce, 2003). Therefore, it is important to know what the social knowledge and demand are. The residents of Sporades consider that the most important activities in the short-term, emergency stage of relief, according to residents' opinions, were the operations of search and rescue of the victims, the medical care of the injured, the water supply and the transportation of people to safer places.

Also, to improve the effectiveness of residents' compliance with warning and evacuation messages, it is important that emergency management officials understand how the public interprets their situation in relation to hazards, and their potential response during a crisis, and apply this information to the ongoing development of risk mitigation strategies (Bird et al., 2009).

The concept of agreement on objectives is potentially able to lower the given disaster risk by bringing together the actors involved throughout the disaster cycle and improving the inter-organizational coordination (Greiving et al., 2012). For that, the risk management should not be the exclusive 
Table 7. Residents' opinions about the activities that take place in the Sporades islands regarding the prevention of forest fires.

\begin{tabular}{|c|c|c|c|c|c|c|}
\hline & & Very often & Usually & Sometimes & Rarely & Never \\
\hline Fire service often conducts frequent patrols in the forests & $s_{\mathrm{p}}$ & 0.0241 & 0.0244 & 0.0206 & 0.0118 & 0.0134 \\
\hline The municipality and the volunteers patrol in the forests & $s_{\mathrm{p}}$ & 0.0212 & 0.0203 & 0.0211 & 0.0202 & 0.0194 \\
\hline There are fire guardrooms during the summer season & $p$ & $26.0 \%$ & $23.6 \%$ & $19.6 \%$ & $17.7 \%$ & $13.1 \%$ \\
\hline \multirow[t]{2}{*}{ Citizens light fires to burn agricultural remains } & $p$ & $25.2 \%$ & $20.6 \%$ & $29.8 \%$ & $18.8 \%$ & $5.6 \%$ \\
\hline & $s_{\mathrm{p}}$ & 0.0225 & 0.0209 & 0.0234 & 0.0198 & 0.0119 \\
\hline \multirow[t]{2}{*}{ Citizens throw lit cigarettes from their cars } & $p$ & $17.4 \%$ & $21.4 \%$ & $35.4 \%$ & $23.9 \%$ & $1.9 \%$ \\
\hline & $s_{\mathrm{p}}$ & 0.0195 & 0.0212 & 0.0243 & 0.0221 & 0.0070 \\
\hline Citizens participate in the suppression of fires & $p$ & $41.8 \%$ & $29.5 \%$ & $18.5 \%$ & $9.4 \%$ & $0.8 \%$ \\
\hline \multirow[t]{2}{*}{ The municipality removes vegetation from the edges of roads and paths } & $p$ & $13.9 \%$ & $23.1 \%$ & $30.8 \%$ & $21.4 \%$ & $10.7 \%$ \\
\hline & $s_{\mathrm{p}}$ & 0.0178 & 0.0212 & 0.0238 & 0.0211 & 0.0158 \\
\hline \multirow[t]{2}{*}{ Citizens remove dried vegetation from their property } & $p$ & $11.5 \%$ & $28.4 \%$ & $36.2 \%$ & $17.4 \%$ & $6.4 \%$ \\
\hline & $s_{\mathrm{p}}$ & 0.0166 & 0.0233 & 0.0244 & 0.0196 & 0.0127 \\
\hline \multirow[t]{2}{*}{ There is forest protection } & $p$ & $20.1 \%$ & $28.4 \%$ & $31.9 \%$ & $13.4 \%$ & $6.2 \%$ \\
\hline & $s_{\mathrm{p}}$ & 0.0203 & 0.0232 & 0.0236 & 0.0175 & 0.0125 \\
\hline \multirow[t]{2}{*}{ There is illegal occupation on forestlands } & $p$ & $27.9 \%$ & $23.3 \%$ & $21.7 \%$ & $14.5 \%$ & $12.6 \%$ \\
\hline & $s_{\mathrm{p}}$ & 0.0227 & 0.0220 & 0.0212 & 0.0178 & 0.0169 \\
\hline
\end{tabular}

responsibility of one sole organization; rather, it should be the result of coordinated actions from many organizations, where everyone has a specific role in the grid of complex activities that is required for the confrontation of the emergency situation. Namely, engagement in the decision-making process, while managing risk, is not only a responsibility of scientists and local authorities, but also the duty of the people that live in the exposed region.

According to Friedmann (1992), people in their own communities have to take their destiny into their own hands, the community should determine its own future, individual and collective needs must be balanced and there must be a move towards self-reliance. As Aguirre (1994) indicates, choosing the best way to engage and involve the public is critical, as is instilling in them a sense of individual responsibility via disaster preparedness. Furthermore, case studies that encourage full participation from the community from the outset appear to be the most sustainable, and addressing underlying causes of vulnerability with active participation of community members and groups can result in sustainable initiatives.

We must learn from our faults. Nevertheless, the management of crisis and disaster, as a newly "recognized" sector, constitutes a great example that we should learn from our mistakes, while it simultaneously offers to us the possibility for major changes and different decisions (Fleischhauer et al., 2012).

The residents believe that, from the point of view of the services, they do not accomplish what should be done to face natural disasters in the best possible manner. Specifically, in the cases of earthquake and forest fire hazards, it was reported that all the activities that are described in the questionnaire should be done very often because the region is seismic and the three islands are covered by dense vegetation. Of course, the latter applies as much to the responsible services as to the same residents.

As for the floods, respondents seemed to think that the illegal occupation of streams and polders occurred very often while the construction and preservation of flood protection works occurs only sometime. Additionally, respondents felt that public roads with drainage systems were only sometimes constructed properly, and a similar response was provided for the consistency with which recently burned forest slopes were stabilized. According to De Graff et al. (2013), empirical models of estimating the probability of erosion and 
landslides can be used to provide critical information for post-fire hazard mitigation and to plan the use of sustainable land management techniques (Panagopoulos and Antunes, 2008). Regarding the snow and frost, respondents felt that they sometimes faced problems of this nature; however, they felt that controls, limitations, and exclusions were rarely in effect and roads were rarely closed due to cars blocking them as a result of these circumstances.

For earthquakes, residents felt that licensing infringements and the construction of illegal buildings occurred very often, while the lack of information of forthcoming earthquakes occurred rarely, thereby confirming Varotsos et al. (2011). The respondents also leaned towards the response of "never" regarding how frequently buildings were checked to determine if they meet earthquake standards, as well as on the provision of information to citizens on the actions that should be taken in case of an earthquake.

Finally, concerning forests and forest fires, the residents declared that the guardhouses are very often manned during the fire season and the citizens participate in the suppression of forest fires, while at the same time also observing illegal occupation on forestlands. Municipality sometimes removes the vegetation from the edges of the roads and the paths and, with the volunteers and fire service, conducts frequent patrols in the forests. On the part of the citizens, it was remarked that citizens sometimes clean the dried vegetation from their property; they also light fires for burning agricultural remains, even during the period when this is prohibited, and they throw lit cigarettes from their cars. They rarely participate in reforestations, while noting that, sometimes, forest protection and forest cleanup were accomplished.

Still, a considerable amount of effort has gone into understanding disaster risks (Alexander, 1997; McGranahan et al., 2001; Pelling, 2003b). The response from participants indicated that much needs to be done to provide a means of enhancing community awareness and uptake of emergency preparedness. The perceptions and attitudes of the residents in the Sporades islands about the management of natural disasters, and their expectations from the authorities and relevant stakeholders, help us to learn from past mistakes and to prepare a pragmatic disaster risk management plan.

Acknowledgements. The authors want to thank the support of Research Center for Spatial and Organizational Dynamics (CIEO), Fundação para a Ciência e a Tecnologia for having provided the conditions to publish this work.

Edited by: M. Parise

Reviewed by: two anonymous referees

\section{References}

Aguirre, B.: Planning, Warning, Evacuation and Search and Rescue: A Review of the Social Science Research Literature, Recovery Center Texas, A\&M University, Texas, 1994.

Alexander, D.: The study of natural disasters, 1977-1997: some reflections on a changing field of knowledge, Disasters, 21, 284304, 1997.

Alexander D.: An evaluation of medium-term recovery processes after the 6 April 2009 earthquake in Aquila, Central Italy, Environ. Hazards, 12, 60-73, 2013.

Amini Hosseini, K., Kazem Jafari, M., Hosseini, M., Mansouri, B., and Hosseinioon, S.: Development of urban planning guidelines for improving emergency response capacities in seismic areas of Iran, Disasters, 33, 645-664, 2009.

Armas, J. and Avram, E.: Perception of flood risk in Danube Delta, Romania, Nat. Hazards, 50, 269-287, 2009

Becker, J., Paton, D., Johnston, D., and Ronan, K.: A model of household preparedness for earthquakes: how individuals make meaning of earthquake information and how this influences preparedness, Nat. Hazards, 64, 107-137, 2012.

Becker, J., Paton, D., Jonston, D., and Ronan, K.: Salient beliefs about earthquake hazards and household preparedness, Risk Anal., 33, 1710-27, 2013.

Bird, D. K., Gisladottir, G., and Dominey-Howes, D.: Resident perception of volcanic hazards and evacuation procedures, Nat. Hazards Earth Syst. Sci., 9, 251-266, doi:10.5194/nhess-9-2512009, 2009.

Boulle, P., Vrolijks, L., and Palm, E.: Vulnerability reduction for sustainable urban development, J. Counting Crisis Manag., 5, 179-188, 1997.

Bowman, L. and White P.: "Community" risk perceptions of a disaster risk reduction intervention at Santa Ana (Ilamatepec) Volcano, El Salvador, Environ. Hazards, 11, 138-154, 2012.

Christoplos, I., Mitchell, J., and Liljelund, A.: Re-framing risk: the changing context of disaster mitigation and preparedness, Disaster 25, 185-198, 2001.

Cutter, L. S., Boruff, J. B., and Shirley, W. L.: Social vulnerability and environmental hazards. Soc. Sci. Quart., 84, 242-261, 2003.

Daoutopoulos, G.: Methodology in Social and Rural Science, Thessaloniki, 1990 (in Greek).

De Graff, J. V., Cannon, S. H., and Parise, M.: Limiting the immediate and subsequent hazards associated with wildfires. In: Margottini C., Canuti P. \& Sassa K. (Eds.), Landslide science and practice, Volume 4: Global Environmental Change. Springer, 199-209, 2013.

Djalante, R.: Review Article: "Adaptive governance and resilience: the role of multi-stakeholder platforms in disaster risk reduction", Nat. Hazards Earth Syst. Sci., 12, 2923-2942, doi:10.5194/nhess-12-2923-2012, 2012.

Drabek, T. E.: Human System Responses to Disaster: An Inventory of Sociological Findings, Springer-Verlag, New York, 1986.

EM-DAT: Annual Disaster Statistical Review: The Numbers and Trends, Centre for Research on the Epidemiology of Disasters (CRED), Universite Catholique de Louvain, Brussels, 2010.

EM-DAT: The OFDA/CRED International Disaster Database, Universite Catholique de Louvain, Brussels, 2013.

Escuder-Bueno, I., Castillo-Rodriguez, J., Zechner, S., Jobstl, C., Perales-Momparler S., and Petaccia, G.: Aquantitative flood risk analysis methodology for urban areas with integration of so- 
cial research data, Nat. Hazards Earth Syst. Sci., 12, 2843-2863, doi:10.5194/nhess-12-2843-2012, 2012.

Fleischhauer, M., Greiving, S., Flex, F., Scheibel, M., Stickler, T., Sereinig, N., Koboltschnig, G., Malvati, P., Vitale, V., Grifoni, P. and Firus, K.: Improving the active involvement of stakeholders and the public in flood risk management - tools of an involvement strategy and case study results from Austria, Germany and Italy, Nat. Hazards Earth Syst. Sci., 12, 2785-2798, doi:10.5194/nhess-12-2785-2012, 2012.

Friedmann, J.: Empowerment: The Politics of Alternative Development, Blackwell, Cambridge, MA, 1992.

Germanwatch: Global Climate Risk Index 2010, Germanwatch, Berlin, available at: http://www.germanwatch.org/klima/cri2010. pdf (last access: 2 July 2010), 2010.

Gero, A., Meheux, K., and Dominey-Howes, D.: Integrating community based disaster risk reduction and climate change adaptation: examples from the Pacific, Nat. Hazards Earth Syst. Sci., 11, 101-113, doi:1.5194/nhess-11-101-2011, 2011.

Greiving, S., Pratzler-Wanczura, S., Sapountzaki, K., Ferri, F., Grifoni, P., Firus, K. and Xanthopoulos, G.: Linking the actors and policies throughout the disaster management cycle by "Agreement on Objectives" - a new output-oriented management approach, Nat. Hazards Earth Syst. Sci., 12, 1085-1107, doi:10.5194/nhess-12-1085-2012, 2012.

Haimes, Y. Y.: Strategic preparedness for recovery from catastrophic risks to communities and infrastructure systems of systems, Risk Anal., 32, 1834-1845, 2012.

IFRC: World Disaster Report 2010, International Federation of Red Cross and Red Crescent Society, available at: http://www.ifrc. org/ (last access: 8 July 2010), 2010.

IPCC: Climate Change 2007: Synthesis Report, Intergovernmental Panel on Climate Change, available at: http://www.ipcc.ch/pdf/ assessment-report/ar4/syr/ar4_syr.pdf (last access: 8 November 2011) 2007.

Khanm, S.: Disasters contributions of hazardscape and gaps in response practices. Nat. Hazards Earth Syst. Sci., 12, 3775-3787, doi:10.5194/nhess-12-3775-2012, 2012.

Lekkas, E.: Natural and Technological Disasters, available at: http://labtect.geol.uoa.gr/pages/lekkase/PDF \{\%\}20Files/ fysikes_katastrofes.pdf (last access: 7 July 2011), 2000.

Maplecroft,: Natural Disaster Risk Index 2010, Maplecroft, London, UK, 2010.

McEntire, A. D.: Development, disaster and vulnerability: a discussion of divergent theories and the need for their integration, Disaster Prev. Manag., 13, 193-198, 2004.

McGranahan, G., Jacobi P., Songsore J., Surjadi C., and Kjellen M.: The citizens at risk: from urban sanitation to sustainable cities, Earthscan Publications Ltd., UK, 2001.

Mendelsohn, R. and Dinar, A.: Climate change, agriculture, and developing countries: does adaptation matters, World Bank Res. Obser., 14, 277-293, 1999.

Mercer, J., Kelman I., Suchet-Pearson, S., and Lloyd, K.: Integrating indigenous and scientific knowledge bases for disaster risk reduction in Papua New Guinea, Geogr. Ann. B, 91, 157-183, 2009.

Otero, R. C. and Marti, R. Z.: The impacts of natural disasters on developing economies: implications for the international development and disaster community, in: Disaster Prevention for Sustainable Development: Economic and Policy Issues, edited by:
Munasinghe, M. and Clarke, C., The World Bank and International Decade for Natural Disaster Reduction, Washington, 1995.

Papadopoulos, G. A.: The Civil Protection in Greece - Confronting Physical and technical disasters, Ion Editions, Athens, Greece, 2000.

Panagopoulos, T. and Antunes, M. D. C.: Integrating geostatistics and GIS for assessment of erosion risk on low density Quercus suber woodlands of South Portugal, Arid Land Res. Manag., 22, 159-177, 2008.

Pearce, L.: Disaster management and community planning, and public participation: how to achieve sustainable hazard mitigation, Nat. Hazards, 28, 211-228, 2003.

Pelling, M.: The Vulnerabilities of Cities: Natural Disasters and Social Resilience, Earthscan Publications Ltd., UK, 2003a.

Pelling, M.: Natural Disasters and Development in a Globalizing World, Routledge, New York, NY, 2003b.

Raschky, P. A.: Institutions and the losses from natural disasters, Nat. Hazards Earth Syst. Sci., 8, 627-634, doi:10.5194/nhess-8627-2008, 2008.

Ravindranath, N. H. and Sathaye J. A.: Climate Change and Developing Countries, Kluwer Academic Publishers, London, 2002.

Salter, J.: Risk management in a disaster management context, J. Counting Crisis Management, 5, 60-65, 1997.

Shi, P.: On the role of government in integrated disaster risk governance-based on practices in China, Int. J. Disaster Risk Sci., 3, 139-146, 2012.

Starmer, C. V.: Explaining risky choices without assuming preferences, Soc. Choice Welfare, 13, 201-213, 1996.

Stenchion, P.: Development and disaster management, Aust. J. Emerg. Manag., 12, 40-44, 1997.

Sterlacchini, S., Frigerio, S., Giacomelli, P., and Brambilla, M.: Landslde risk analysis: a multi-disciplinary methological approach, Nat. Hazards Earth Syst. Sci., 7, 675-675, doi:10.5194/nhess-7-657-2007, 2007.

UNDP: Reducing Disaster Rsk a Challenge for Development: a Global Report, United Nations Development Program, New York, 2004.

UNISDR: Global Assessment Report on Disaster Risk Reduction: Revealing Risk, Redefining Development, United Nations Publications, UK, available at: http://www.preventionweb.net/files/ globalplatform/5198f8ce8fe5bExecutive_Summary_Revealing Risk,_Redefining_Development._Global_Assessment_Report_ on_Disaster_Risk_Reduction_2011.pdf (last access 8 September 2011), 2011.

UNU-EHS: World Risk Report 2011, United Nations University, Institute for Environment and Human Security, Bonn, available at: http://www.ehs.unu.edu/file/get/9018 (last access 6 June 2011), 2011.

Van Assche, K., Duineveld, M., Beunen, R., and Teampau, P.: Delineating locals: transformations of knowledge/power and the governance of the Danube Delta, J. Environ. Pol. Plan., 13, 121, 2011.

Varnes, D. J.: Landslide Hazard Zonation: A Review of Principles and Practice, UNESCO, Paris, 1988.

Varotsos, P., Sarlis, N., and Skordas, E.: Natural Time Analysis: the New View of Time; Precursory Seismic Electric Signals, Earthquakes and Other Complex Time Series, Springer, Berlin, 2011. 
Whitworth, P. and May, F.: Disaster reduction planning for recreation areas via cascading models. J. Park Recr. Admin., 24, available at: http://js.sagamorepub.com/jpra/article/view/1392, 2006.

Winkler, H.: Climate change and developing countries, S. Afr. J. Sci., 101, 355-364, 2005.

Wisner, B., Blaikie, P., Cannon, T., and Davis, I.: At Risk: Natural Hazards, Peoples Vulnerabilities and Disasters, Routledge, New York, 2004
Ye, M. W., Wang, J., Huang J., Xu, S., and Chen, Z.: Methodology and its application for community-scale evacuation planning against earthquake disaster. Nat. Hazards, 61, 881-892, 2012.

Yellman, W. T. and Murray, M. M.: Vulnerability and resilience. Risk Anal., 33, 753 pp., 2013. 